DR YANIRA RIFFO-VASQUEZ (Orcid ID : 0000-0002-2137-9430)

DR FRANCIS MAN (Orcid ID : 0000-0002-5076-2180)

Article type : Original Article-Experimental Models of Allergic Disease

\title{
MODULATION OF ALLERGIC INFLAMMATION IN THE LUNG BY A PEPTIDE DERIVED FROM MYCOBACTERIA TUBERCULOSIS CHAPERONIN 60.1
}

Running Title: Modulation of allergic lung inflammation by a bacterial derived peptide

\author{
Yanira Riffo-Vasquez ${ }^{1}$, Varsha Kanabar ${ }^{1}$, Sandra D. Keir ${ }^{1}$, Rodrigo R. e-Lacerda ${ }^{1}$, Francis \\ Man', David J. Jackson ${ }^{2,3}$, Valerie Corrigall ${ }^{1}$, Anthony R.M. Coates ${ }^{4}$ and Clive P. Page ${ }^{1}$.
}

${ }^{1}$ Sackler Institute of Pulmonary Pharmacology, Institute of Pharmaceutical Sciences, King's College London, 150 Stamford Street, London, SE1 9NH, UK. ${ }^{2}$ Asthma Care Guy's \& St Thomas' NHS Trust and ${ }^{3} \mathrm{MRC} \&$ Asthma UK Centre, Faculty of Life Sciences and Medicine $\mid 5^{\text {th }}$ Floor Bermondsey Wing, Guy's Hospital, King's College London, London, SE1 9RT, UK. 'Medical Microbiology, Institute of Infection and Immunity, St George's, University of London, Cranmer Terrace, London, SW17 0RE, UK.

\section{Word count: 3,963}

Table count: 0

Figures count: 8

Correspondence and requests for reprints should be addressed to: Yanira Riffo-Vasquez, King's College London. 150 Stamford Street, London SE1 9NH, UK. yanira.vasquez@kcl.ac.uk, Orcid 0000002-2137-9430. Phone: +442078484819 , FAX: +442078484788

This article has been accepted for publication and undergone full peer review but has not been through the copyediting, typesetting, pagination and proofreading process, which may lead to differences between this version and the Version of Record. Please cite this article as doi: $\underline{10.1111 / \mathrm{CEA} .13550}$

This article is protected by copyright. All rights reserved 


\section{Abstract:}

Background: We have previously demonstrated that M. tuberculosis chaperonin 60.1 inhibits leukocyte diapedesis and bronchial hyperresponsiveness in a murine model of allergic lung inflammation. Methods: In the present study we have investigated the effect of a shorter peptide sequence derived from Cpn 60.1, named IRL201104, on allergic lung inflammation induced by ovalbumin (OVA) in mice, and by house dust mite (HDM) in guinea pigs, as well as investigating the action of IRL201104 on human cells in vitro. Results: Pre-treatment of mice or guinea pigs with IRL201104 inhibits the infiltration of eosinophils to the lung, cytokine release, and in guinea pig skin, inhibits allergen-induced vascular permeability. The protective effect of intra-nasal IRL201104 against OVA-induced eosinophilia persisted for up to 20 days post treatment. Moreover, OVAsensitized mice treated intra-nasally with $20 \mathrm{ng} / \mathrm{kg}$ of IRL201104 show a significant increase in the expression of the anti-inflammatory molecule ubiquitin A20, and significant inhibition of the activation of $\mathrm{NF}-\kappa \mathrm{B}$ in lung tissue. Our results also show that A20 expression was significantly reduced in blood leukocytes and ASM obtained from patients with asthma compared to cells obtained from healthy subjects which was restored after incubation with IRL201104 in vitro, when added alone, or in combination with LPS or TNF- $\alpha$ in ASM. Conclusions: Our results suggest that a peptide derived from mycobacterial Cpn60.1 has a long-lasting anti-inflammatory and immunomodulatory activity which may help explain some of the protective effects of TB against allergic diseases.

This article is protected by copyright. All rights reserved 


\section{Introduction}

Heat Shock Proteins of the HSP60 family are also called chaperonins and form a structurally related subfamily of molecular chaperones. They can be classified into Group I and Group II chaperonins: Group I comprises chaperonins found in bacteria, mitochondria and chloroplasts, whereas Group II chaperonins are found in the cytosol of eukaryotes and in archaea ${ }^{1}$. Group I chaperonins are divided into Chaperonin 60 (Cpn60) and Chaperonin 10 (Cpn10) proteins, according to the approximate molecular weight (in $\mathrm{kDa}$ ) of their subunits. However, M. tuberculosis and M. bovis encode a second chaperonin 60 , named chaperonin $60.1^{2}$.

$\mathrm{Hu}$ et al. demonstrated that the Cpn60.1 gene is not essential for M. tuberculosis survival. Furthermore, growth and proliferation of the mycobacteria in culture and in mouse lungs were not affected by the absence of Cpn60.1. However, the mutant strain of M. tuberculosis did not elicit the expected granulomatous inflammation in the lungs of mice following and that would normally be observed following infection with the wild-type strain. Such observations suggest that Cpn60.1 plays an important role in granuloma formation following infection with tuberculosis ${ }^{3}$.

A number of epidemiological studies have suggested an inverse correlation between allergic asthma and vaccination against tuberculosis with the attenuated form of the bacteria, the bacillus CalmetteGuerin $(\mathrm{BCG})^{4-7}$. Data from the literature reveal an inverse correlation between the incidence of tuberculosis and the development of allergic diseases like rhinitis or eczema ${ }^{8,9}$. More importantly, patients exposed to M. tuberculosis are less likely to develop asthma later in life, strongly suggesting that some component(s) of mycobacteria must have a protective effect against allergy and asthma ${ }^{10}$, 11 .

The traditional view of molecular chaperones is that of proteins which mediate the folding of other proteins and peptides ${ }^{12}$. However, over the past 20 years, evidence has appeared that some chaperone proteins have distinct, additional roles, notably as intercellular signaling molecules in the immune system. It has also been demonstrated that Cpn60.1 at high concentrations is a potent inducer of inflammatory cytokines, including IL-6, IL-8, IL-12, TNF- $\alpha$ and GM-CSF, and the monocytedeactivating cytokine IL-10 from human peripheral monocytes in vitro ${ }^{13}$, as well as IL-1 and IL-6 from murine macrophages ${ }^{14}$.

This article is protected by copyright. All rights reserved 
We have previously demonstrated that the chaperonin 60.1 (Cpn60.1) derived from $M$. tuberculosis inhibits leukocyte diapedesis, pulmonary eosinophilia, bronchial hyperresponsiveness and associated increases in pro-inflammatory cytokines in a mouse model of allergic lung inflammation ${ }^{15,16}$. In the present study therefore, we have investigated the effect of a shorter sequence of M. tuberculosis Cpn60.1, named IRL201104, in an attempt to identify the biologically active sequence of this chaperone responsible for manipulating the allergic inflammatory response ${ }^{17}$. We have investigated the effect of IRL201104 on allergic lung inflammation induced by OVA in mice, or induced by HDM in guinea pigs, as well as investigating the action of IRL201104 on human leukocytes and ASM cells in vitro.

This article is protected by copyright. All rights reserved 


\section{Materials and Methods}

Further detailed methods can be found in the supplemental section

\section{OVA-Induced Allergic Inflammation in mice}

Female BALB/c mice (Envigo, UK), were immunized with $30 \mu \mathrm{g}$ of OVA i.p. (type V; Sigma Chemical Co., Gillingham, UK) adsorbed to aluminum hydroxide, as described previously ${ }^{16}$. On day 14 of sensitization, all mice were challenged with a 3\% aerosol of OVA, once a day for 3 consecutive days. Experiments were approved by the Home Office under "The Animals (Scientific Procedures) Act (1986)" and by the Ethics Committee of King's College London.

\section{Bronchoalveolar lavage in mice}

Twenty four hours after last OVA challenge, mice were anaesthetized with urethane $(2 \mathrm{~g} / \mathrm{kg}$ i.p.; Sigma Gillingham, UK.), a cannula inserted into the exposed trachea and three $0.5 \mathrm{~mL}$ aliquots of sterile saline were injected into the lungs. The total number of cells in the lavage fluid was counted with an improved Neubauer hemocytometer. For differential cell counts, cytospin preparations were stained with Diff Quick (DADE Behring, Switzerland) and cells were counted using standard morphological criteria

\section{Treatment of mice}

IRL201104 corresponds to residues 461-491 in the equatorial domain of Cpn60.1 ${ }^{17}$ (kindly provided by Immune Regulation Ltd, Stevenage, UK) and was prepared by diluting in sterile saline with $0.1 \%$ BSA (bovine serum albumin) and administered intranasally (2-200 $\mathrm{ng} / \mathrm{kg}$ ) or intravenously $(20 \mathrm{ng} / \mathrm{kg})$ before each OVA challenge in a total of 3 treatments. Controls received the vehicle only.

\section{Cytokine Levels}

Levels of IL-5 and IL-12 were measured in BAL fluid by standard ELISA (eBioscience, Hatfield, UK) following instructions from the manufacturer. 


\section{A20 and NF- $\kappa$ B expression measured by immunohistochemistry in mice}

Mouse anti A20 (bs-2803R, Bioss Antibodies, USA), NF-кB p65 (14-6731-81, Invitrogen, UK) or phosphorylated NF- $\mathrm{B}$ (ab86299, Abcam, UK) were used for the immunohistochemistry experiments. Quantification was performed using Image-Pro Plus software, v5.5.0.29 (Media Cybernetics, USA).

\section{HDM-induced Allergic Inflammation in guinea pigs}

Immunizations were performed with $50 \mu \mathrm{g}$ of House Dust Mite (HDM), Dermatophagoides pteronyssinus, (Greer Laboratories Inc., Charlotte, USA), adsorbed to a saturated solution of aluminum hydroxide s.c. (Sanofi Ltd., Sao Paulo, Brazil). On day 15 animals were challenged with 50 $\mu \mathrm{g}$ HDM intra-tracheally. Experiments were approved by the UK Home Office under "The Animals (Scientific Procedures) Act (1986)" and by the Ethics Committee of King's College London.

\section{Administration of IRL201104 to guinea pigs}

IRL201104 was diluted in sterile saline with 0.1\% BSA (bovine serum albumin) and administered intravenously $(0.3 \mathrm{~mL} ; 200 \mathrm{ng} / \mathrm{kg} ; 80 \mathrm{ng}$ per guinea pig weighing $400 \mathrm{~g})$ immediately prior to HDM challenge on day 15. Controls received vehicle only. Skin testing and BAL was performed 24h later.

\section{Skin tests and Bronchoalveolar lavage in guinea pigs}

Evans Blue dye was injected intravenously $(0.5 \mathrm{~mL})$ followed immediately by intradermal injections of saline, HDM 1:5, 1:50 or 1:500 dilutions from the stock solution of HDM containing 1 $\mathrm{mg} / \mathrm{mL}$ protein. One hour later, BAL was performed, and treated skin samples removed, weighed and placed in formamide. Optical density of the BAL fluid was measured at $620 \mathrm{~nm}$.

\section{Lung Function in Guinea Pigs}

Guinea pigs were ventilated $(8 \mathrm{~mL} / \mathrm{kg} ; 60$ breaths $/ \mathrm{min})$ through a tracheal cannula connected to a pneumotachograph and a pressure transducer $\left( \pm 2 \mathrm{~cm} \mathrm{H}_{2} \mathrm{O}\right.$ : model MP-45-14-871; Validyne Engineering, Northridge, CA, U.S.A.). Changes in airflow were measured using an automated lung 
function recording system (Pulmonary Monitoring System, version 5.0; Mumed, London, U.K.) Airway obstruction was measured [as an increase in $R_{L}$ and decrease in lung compliance $\left(C_{d y n}\right)$ ] immediately after administration of $100 \mu \mathrm{g} / \mathrm{kg}$ of HDM i.v.

\section{Experiments with human cells}

Ethical approval for the biopsies collected from healthy and asthmatic subjects was provided by Guy's and St Thomas' Hospitals Research Ethics Committee (REC: 08/H0804/67). The blood collection from healthy and asthmatic subjects was approved by the National Research Ethics Committee at Guy's and St Thomas' Hospitals (10/H0807/99). Specific demographics regarding these patients can be found in the Supplemental Material and Methods Section. Appropriate consents, permissions and releases were obtained prior collection of all human samples.

\section{Isolation and culture of Blood leukocytes}

Whole blood from healthy or asthmatic volunteers was collected and some was immediately stained for TNFAIP3(A20) detection by flow cytometry. The remainder was diluted 1:1 with RPMI 1640 supplemented with glutamine (Invitrogen, Paisley, UK), and cultured with or without IRL201104 $(0.1-10 \mu \mathrm{g} / \mathrm{mL})$. After $24 \mathrm{hr}$ of incubation, cells were harvested for intracellular A20 expression analysis by flow cytometry.

\section{A20 detection by flow cytometry}

Following treatments, cells were harvested and fixed, permeabilized and incubated with PerCP conjugated rabbit anti-human A20/TNFAIP3 (1:50, clone EPR2663, ab220511, Abcam, UK). 10,000100,000 events in a leukocyte gate were collected and analyzed using a Beckman FC500 flow cytometer.

\section{A20 expression detected by immunohistochemistry in human lung tissues}

Frozen human biopsies were incubated with anti-human A20 antibody (1:100, clone 775928MAB75981, R\&D, UK). Expression was quantified using Image-Pro Plus software, v5.5.0.29. 


\section{Preparation of human airway smooth muscle}

ASM was isolated from the biopsies obtained from subjects with asthma and healthy subjects, and seeded at 5,000 cells $/ \mathrm{cm}^{2}$ and allowed to adhere to glass for $24 \mathrm{~h}$. ASM were then growth-arrested in serum-free DMEM (which also served as a control) for $24 \mathrm{~h}$ and then treated with IRL201104 (0.5$5 \mathrm{pg} / \mathrm{mL})$.

\section{A20 detection by immunofluorescence in human ASM}

Treated ASM were incubated with anti-human A20 (MAB75981, R\&D Systems). Fluorescence was quantified using Image J, v 1.45s (NIH, USA).

\section{Statistical analysis}

Data are expressed as mean \pm SEM or as median with 95\% CI. Comparisons were made by one-way ANOVA followed by appropriate post-hoc test or t-test using GraphPad Prism (version 7.0). The specific post-hoc test applied in each set of experiments is described in the legend of the figures. 


\section{Results}

\section{IRL201104 inhibits acute allergic lung inflammation induced by OVA in sensitized mice}

Our results confirm that immunization and challenge with OVA induce a significant increase in the total number of leukocytes in BAL fluid, reflected by significantly higher numbers of eosinophils in comparison to sham-immunized animals $24 \mathrm{~h}$ after the last OVA challenge (Fig. 1A and B). Pre-treatment with IRL201104 significantly inhibited the increase of total leukocytes and eosinophil numbers at doses of 20 or $200 \mathrm{ng} / \mathrm{kg}$ (Fig. 1A and B). Our results also show that intra-nasal pre-treatment with $20 \mathrm{ng} / \mathrm{kg}$ of IRL201104 also significantly inhibited the release of IL-5 and decreased the levels of IL-12 in BAL fluid $24 \mathrm{~h}$ following the last allergen challenge, suggesting that the anti-inflammatory effect of the peptide is not due to a shift from a Th2 to Th1 cytokine profile (Fig. $1 \mathrm{C}$ and D).

\section{IRL201104 inhibits acute allergic lung inflammation induced by OVA independently of the} route of administration

In order to assess whether the anti-inflammatory effect of IRL201104 is dependent on the route of administration, we chose a dose $(20 \mathrm{ng} / \mathrm{kg})$ of peptide that was significantly effective in inhibiting eosinophil migration when administered via the intra-nasal route and investigated whether the peptide was still effective when given systemically. Our results show that intravenous pretreatment with IRL201104 also significantly inhibited the allergen-induced increase in eosinophil numbers in the BAL fluid at a dose of $20 \mathrm{ng} / \mathrm{kg}$ (Fig. 1E and F).

\section{IRL201104 inhibits acute allergic lung inflammation independently of the allergen or the species}

Our results show that pre-treatment of guinea pigs with IRL201104 (200 ng/kg intravenously) significantly prevented the increase in the number of eosinophils in BAL fluid $24 \mathrm{~h}$ after a single intra-tracheal challenge with $50 \mu \mathrm{g}$ of HDM (Fig. 2A). In addition, pre-treatment with IRL201104 also inhibited the extravasation of Evans blue dye into the skin measured 1 hour after the intradermal injection of diluted HDM in saline at ratios of 1:5 and 1:50 in guinea pig skin (Fig. 2B). More 
importantly, we also demonstrated that IRL201104 significantly prevented the increase in airways resistance induced by a single challenge with HDM (100 $\mu \mathrm{g} / \mathrm{kg}$, i.v) (Fig. 2C).

\section{The time course of the effect of IRL201104 on allergic lung inflammation}

We treated OVA-sensitized mice intravenously or intranasally just before each OVA challenge with the lowest effective concentration of IRL201104, $20 \mathrm{ng} / \mathrm{mouse}$. Ten, 20 or 30 days later, groups of these animals were challenged again with OVA for 3 consecutive days without further treatments with IRL201104. A schematic representation of the protocol of sensitization and treatment can be found in the Supplementary Material accompanying this manuscript (Supplemental Figure 1).

Our results confirm that IRL201104 inhibits the recruitment of leukocytes to the lung measured $24 \mathrm{~h}$ after the last OVA challenge whether administered intranasally or intravenously (Fig. 3A). Ten days later, intranasal treatment still showed a significant effect on eosinophil recruitment when compared to vehicle-treated animals (Fig. 3B). Twenty days later, the response to OVA was not as robust as seen at earlier time points, even in vehicle-treated animals, but nevertheless there remained a significantly smaller leukocyte infiltration induced by OVA challenge in the animals that had been pretreated intranasally with IRL201104 (Fig. 3C). At 30 days following treatment with IRL201104, the eosinophilic response following OVA challenge was not significantly different from that observed in the vehicle-treated animals receiving OVA (Fig. 3D).

\section{IRL201104 inhibits allergen-induced eosinophil infiltration into the lungs of animals that had previously undergone allergen exposure}

Mice were sensitized and challenged with OVA following the same protocol as described above. One group of mice then received a single dose of $200 \mathrm{ng} / \mathrm{kg}$ of IRL201104 intranasally $24 \mathrm{~h}$ after the last OVA challenge. A second group was intranasally treated with $200 \mathrm{ng} / \mathrm{kg}$ of IRL201104, 24, 48 and $72 \mathrm{~h}$ after the last OVA challenge, in a total of 3 treatments. Ten days after the last OVA challenge, all groups were challenged again with OVA for 3 consecutive days. No further treatments with IRL201104 were performed at these later time points. Twenty-four hours after the $6^{\text {th }}$ challenge, the number of leukocytes were enumerated in the BAL. A schematic representation of the protocol of 
sensitization and treatment can be found in the Supplementary Material accompanying this manuscript (Supplemental Figure 2).

Our results show that regardless of the number of treatments, IRL201104 administered after the third OVA challenge significantly prevented the increase of total number of leukocytes in the lung following further OVA challenges, 10 days later (Fig. 4A). This reduction in allergen sensitivity in IRL201104 treated animals is reflected in a significant inhibition of the number of eosinophils in the BAL fluid obtained from mice treated with IRL201104 once or 3 times after the challenge phase with OVA (Fig. 4B).

\section{IRL201104 modulates the expression of A20 and inhibits the activation of NF- $\mathrm{KB}$ in the lungs of mice sensitized and challenged with OVA}

Considering the bacterial origin of IRL201104, we investigated whether this peptide exerts any of its anti-inflammatory or immunomodulatory effects by a A20/TNFAIP3 mediated pathway. We analyzed the expression of A20 in the lung tissue of mice sensitized and challenged with OVA, as well as the expression of total and activated NF- $\mathrm{BB}$ in these lung samples. Our results show that expression of A20 is reduced in the lungs of untreated OVA-sensitized mice, whilst pre-treatment with IRL201104 significantly prevented this decrease in expression of A20 in the lung tissue (Fig. 5A). More importantly, the effect of IRL201104 on A20 expression paralleled the time course of the effect of the peptide on eosinophil numbers in the BAL fluid (Figure 3). In addition, we have also shown that whilst IRL201104 does not alter the expression of total NF- $\mathrm{B}$ in the lungs of OVAsensitized and challenged mice, it does inhibit the activation of this transcription factor (Fig. 5B and C).

\section{IRL201104 induces the expression of A20 in human cells}

We quantified the expression of A20 in lung biopsies and circulating leukocytes collected from healthy and asthmatic donors. Expression of A20 was measured in lung tissue by immunohistochemistry and in circulating leukocytes by flow cytometry. Results are presented in Figure 6 and we have demonstrated a smaller area of positive expression for A20 in lungs from mild asthmatic patients in comparison to tissue obtained from healthy subjects (Fig. 6A and C). Similarly, 
we observed a significantly lower expression of A20 in leukocytes obtained from severe asthmatic donors in comparison to healthy subjects (Fig. 6C and D, supplemental figure 4).

In the next part of our study, we investigated the effect of IRL201104 on the expression of A20 in human circulating leukocytes collected from asthmatic and healthy donors. A20 expression was measured by flow cytometry in full blood cultures incubated for $24 \mathrm{~h}$ with IRL201104. Our results show that IRL201104 at $0.1 \mu \mathrm{g} / \mathrm{mL}$ had a significant effect on the expression of A20 in leukocytes collected from healthy and asthmatic donors (Figure 7A and B, panels $\mathrm{C}$ and D), (Additional representative scatter plots can be found in supplemental figure 5A and 5B).However, pre-treatment with IRL201104 did not significantly alter the mean fluorescence intensity (MFI) in healthy or asthmatic leukocytes (Supplemental Figure 3).

Given that ASM bundles were found to express A20 in our stained biopsy data (Figure 6A), we also investigated the effect of IRL201104 on the expression of A20 in cultured human ASM which were dissected from the bronchial biopsies collected from asthmatic and healthy donors, and treated in vitro with pro-inflammatory cytokines and stimulant. A20 expression was measured by immunofluorescence in cells incubated for $4 \mathrm{~h}$ with IRL201104 alone, or in combination with TNF- $\alpha$ or LPS. Our results show that IRL201104 at 0.5 and $5 \mathrm{pg} / \mathrm{mL}$ had no significant effect on the expression of A20 in ASM collected from healthy donors (Figure 8A, B and C and representative photographs of healthy cells a-d). In contrast, pre-treatment with IRL201104 alone significantly increased the expression of A20 in ASM isolated from mild asthmatic patients (Fig. 8D), or in combination with $10 \mathrm{ng} / \mathrm{mL}$ of TNF- $\alpha$ (Fig. 8E) or $100 \mathrm{ng} / \mathrm{mL}$ of LPS (Fig. 8F). Photographs e-h represent the fluorescent intensity of A20 in ASM collected from asthmatic subjects.

\section{Discussion}

This article is protected by copyright. All rights reserved 
We have previously reported that the chaperone Cpn60.1 isolated from M. tuberculosis is able to exhibit an important anti-allergic effect by inhibiting allergen-induced eosinophil infiltration into the lungs of sensitized challenged mice and the associated bronchial hyperresponsiveness ${ }^{15}, 16$. In the present study, we have investigated the effect of IRL201104, a shorter peptide sequence isolated from the equatorial domain region of $M$. tuberculosis Cpn60.1, in models of allergic inflammation induced by OVA or HDM ${ }^{17}$. Our results show that pre-treatment of mice or guinea pigs with IRL201104, independently of the route of administration, inhibits the allergic response assessed as a significant inhibitory effect on antigen induced infiltration of eosinophils into BAL fluid, inhibition of allergen induced cytokine release in the lung, with doses of the peptide as low as $20 \mathrm{ng} / \mathrm{kg}$. Additionally, in guinea pigs we also observed inhibition of allergen-induced vascular permeability in skin, and more importantly, airways resistance induced by HDM. As such, our results demonstrate that this peptide has similar anti-inflammatory effects as previously observed with the full $60 \mathrm{kDa}$ chaperonin Cpn60.1 15, 16. In addition, we have demonstrated that one single treatment with IRL201104 administered when allergic inflammation in the lung has been established is sufficient to significantly prevent a second allergic response 10 days later, demonstrating an important and long-lasting benefit of reducing allergic sensitivity way beyond the short half-life of the molecule (less than $10 \mathrm{~min}$, unpublished observations). This disparity between the pharmacokinetic and pharmacodynamic half-life of IRL201104 suggest that this molecule has a profound long-lasting anti-inflammatory activity, possibly by influencing the plasticity of cells involved in the initial allergic response (immune memory or immune resetting).

Recent clinical evidence has suggested that people exposed to "barn dust" in early life are also protected against allergic diseases and asthma 18, 19, an observation supported by the demonstration that mice chronically exposed to low doses of endotoxin-rich environments such as farm dust are protected against the development of allergic lung inflammation in response to HDM by a TLR-4 mediated mechanism ${ }^{20}$. TLR-4 signaling in the lung leads to translocation of NF- $\kappa$ B, increasing the expression of attenuators of this response such as the ubiquitin-modifying enzyme A20 21, 22. The A20 ubiquitin, also known as TNFAIP3, is a zinc-finger protein that attenuates NF- $\mathrm{kB}$ activation by deubiquitinating key signaling pathways downstream of TLR-4, IL-1 and TNF family receptors ${ }^{22-24}$. It is therefore of interest that in OVA-sensitized mice that have been intranasally

This article is protected by copyright. All rights reserved 
treated with $20 \mathrm{ng} / \mathrm{kg}$ of IRL201104, there is a significant increase in the expression of A20 in lung tissue in comparison to untreated OVA-sensitized and challenged mice. Moreover, we observed a close correlation between the effect of IRL201104 on A20 expression and the time course of its protective effect against OVA-induced eosinophil migration into the lung. Analysis of the expression of phosphorylated NF- $\mathrm{NB}$ in the same lung samples demonstrated that the increase in expression of A20 is accompanied by a significant inhibition of the activation of NF- $\kappa B$, suggesting that the antiinflammatory effect of IRL201104 in our model is mediated by A20, similar to results reported with "barn dust" 20.

It has also been shown that A20 expression is reduced in the lungs of subjects with asthma compared with healthy controls ${ }^{20}$. In the present study we have confirmed this observation demonstrating that A20 expression is significantly reduced in lung tissue biopsies, as well as in circulating leukocytes collected from patients with asthma in comparison with healthy subjects, supporting the concept that A20 is an important endogenous modulator of inflammatory responses ${ }^{25}$, 26.

The role of A20 in inflammatory conditions has been well documented. An important antiinflammatory role for this ubiquitin has been reported in arthritis, auto-immune diseases, cardiovascular diseases, cystic fibrosis, chronic obstructive pulmonary disease (COPD), allergies and asthma ${ }^{20,27-33}$. In addition, the modulation of the expression of A20 has been demonstrated to be an important mechanism of action of certain drugs including methotrexate, quercetin and the combination of long-acting $\beta_{2}$-adrenoceptor agonists and glucocorticoids ${ }^{34-37}$. Moreover, it has recently been reported that there is a positive association between a polymorphism in the A20 gene and susceptibility to human asthma in the population investigated in the GABRIELA study, a study that focuses on the effects of farm environments on asthma and allergy in children from central Europe ${ }^{20}$. Furthermore, mRNA levels of A20 measured by quantitative reverse transcriptase polymerase chain reaction (qRT-PCR) were shown to be significantly reduced in epithelial cells obtained from subjects with mild or severe asthma when compared with healthy controls, findings confirmed with protein levels ${ }^{20}$.

In the present study, we collected blood samples from healthy and severe asthmatic subjects and measured the effect of IRL201104 on the expression of A20 in circulating leukocytes after 1 and 
24h of incubation. A one-hour incubation with the peptide did not significantly alter the expression of A20 in circulating leukocytes in comparison to untreated cells (data not shown). However, after $24 \mathrm{~h}$ incubation of whole blood with IRL201104 we observed a significant increase in the expression of A20 in comparison to untreated cells obtained from both asthmatic and healthy subjects, suggesting that IRL201104 can reverse the loss of expression of A20 in circulating leukocytes. Further experiments are necessary to establish the specific blood cell populations affected by IRL201104.

During our analysis of human lung tissue, we observed expression of A20 in the epithelial layer, but we also observed a very significant expression of A20 in ASM bundles (Figure 6). This was an unexpected, but interesting observation as little is known about the role of A20 in ASM in asthma. A previous study has demonstrated, by using RNA-based gene knockdown techniques, that A20 is required for the maximal effect of glucocorticosteroids on cytokine release by human ASM in vitro, indicating that ASM can not only express A20, but that this protein may be important in the modulation of ASM function ${ }^{36}$. In the present study, we also investigated whether cultured human ASM, isolated from healthy or mild asthmatic donors, express A20 and whether this expression can be modulated by IRL201104. Our results show that constitutive levels of A20 expression in healthy ASM, measured by immunohistochemistry, are not significantly altered by treatment with IRL201104, alone or in combination with LPS or TNF- $\alpha$. In contrast, ASM from mild asthmatic donors had a significant increase in the expression of A20 when IRL201104 was added to the culture, alone or in combination with LPS or TNF- $\alpha$. Our results suggest that IRL201104 at very low concentrations can regulate the expression of A20 in ASM from subjects with asthma which may be an important part of the mechanism(s) whereby TB and IRL201104 can inhibit allergic sensitivity.

The results from the present study suggest that IRL201104, a peptide derived from mycobacterial Cpn60.1, can have profound effects on allergen sensitivity that last far beyond the pharmacokinetic half-life of the peptide. These results suggest that this molecule has a long-lasting anti-inflammatory and immunomodulatory activity which may help explain the protective effects of TB against allergic diseases. The observations that this peptide can upregulate A20, shared with the protective effects of barn dust on allergic diseases, provides a plausible common mechanism as to how exposure to bacterial products in early life protects against the development of allergic diseases.

This article is protected by copyright. All rights reserved 
The development of IRL201104 or materials isolated from barn dust as a novel approach to treating allergic diseases appears warranted.

\section{References}

1. Hartl FU, Hayer-Hartl M. Molecular chaperones in the cytosol: from nascent chain to folded protein. Science 2002; 295:1852-8.

2. Coates AR, Shinnick TM, Ellis RJ. Chaperonin nomenclature. Mol Microbiol 1993; 8:787.

3. Hu Y, Henderson B, Lund PA, Tormay P, Ahmed MT, Gurcha SS, et al. A Mycobacterium tuberculosis mutant lacking the groEL homologue cpn60.1 is viable but fails to induce an inflammatory response in animal models of infection. Infect Immun 2008; 76:1535-46.

4. Choi IS, Koh YI. Effects of BCG revaccination on asthma. Allergy 2003; 58:1114-6.

5. Herz U, Gerhold K, Gruber C, Braun A, Wahn U, Renz H, et al. BCG infection suppresses allergic sensitization and development of increased airway reactivity in an animal model. J Allergy Clin Immunol 1998; 102:867-74.

6. Shirakawa T, Enomoto T, Shimazu S-i, Hopkin JM. The Inverse Association Between Tuberculin Responses and Atopic Disorder. Science 1997; 275:77-9.

7. Linehan MF, Frank TL, Hazell ML, Francis HC, Morris JA, Baxter DN, et al. Is the prevalence of wheeze in children altered by neonatal BCG vaccination? Journal of Allergy and Clinical Immunology 2007; 119:1079-85.

8. von Mutius E, Pearce N, Beasley R, Cheng S, von Ehrenstein O, Bjorksten B, et al. International patterns of tuberculosis and the prevalence of symptoms of asthma, rhinitis, and eczema. Thorax 2000; 55:449-53.

9. Obihara CC, Kimpen JL, Gie RP, Lill SW, Hoekstra MO, Marais BJ, et al. Mycobacterium tuberculosis infection may protect against allergy in a tuberculosis endemic area. Clin Exp Allergy 2006; 36:70-6.

10. Shirtcliffe P, Weatherall M, Beasley R. An inverse correlation between estimated tuberculosis notification rates and asthma symptoms. Respirology 2002; 7:153-5.

11. Lighter-Fisher J, Surette AM. Performance of an interferon-gamma release assay to diagnose latent tuberculosis infection during pregnancy. Obstet Gynecol 2012; 119:1088-95.

12. Zhang WJ, Xie HY, Duan X, Wan YL, Peng CH, Shi SH, et al. Study of human B7 homolog 1 expression in patients with hepatitis B virus infection. World J Gastroenterol 2012; 18:3681-95.

13. Lewthwaite JC, Coates AR, Tormay P, Singh M, Mascagni P, Poole S, et al. Mycobacterium tuberculosis chaperonin 60.1 is a more potent cytokine stimulator than chaperonin 60.2 (Hsp 65) and contains a CD14-binding domain. Infect Immun 2001; 69:7349-55.

14. Cehovin A, Coates AR, Hu Y, Riffo-Vasquez Y, Tormay P, Botanch C, et al. Comparison of the moonlighting actions of the two highly homologous chaperonin 60 proteins of Mycobacterium tuberculosis. Infect Immun 2010; 78:3196-206.

This article is protected by copyright. All rights reserved 
15. Riffo-Vasquez Y, Spina D, Page C, Tormay P, Singh M, Henderson B, et al. Effect of Mycobacterium tuberculosis chaperonins on bronchial eosinophilia and hyper-responsiveness in a murine model of allergic inflammation. Clin Exp Allergy 2004; 34:712-9.

16. Riffo-Vasquez Y, Coates AR, Page CP, Spina D. Mycobacterium tuberculosis chaperonin 60.1 inhibits leukocyte diapedesis in a murine model of allergic lung inflammation. Am J Respir Cell Mol Biol 2012; 47:245-52.

17. Hu Y, Coates AR, Liu A, Lund PA, Henderson B. Identification of the monocyte activating motif in Mycobacterium tuberculosis chaperonin 60.1. Tuberculosis (Edinb) 2013; 93:442-7.

18. Riedler J, Braun-Fahrlander C, Eder W, Schreuer M, Waser M, Maisch S, et al. Exposure to farming in early life and development of asthma and allergy: a cross-sectional survey. Lancet 2001; 358:1129-33.

19. Ober C, Sperling AI, von Mutius E, Vercelli D. Immune development and environment: lessons from Amish and Hutterite children. Curr Opin Immunol 2017; 48:51-60.

20. Schuijs MJ, Willart MA, Vergote K, Gras D, Deswarte K, Ege MJ, et al. Farm dust and endotoxin protect against allergy through A20 induction in lung epithelial cells. Science 2015; 349:1106-10.

21. Kelly C, Shields MD, Elborn JS, Schock BC. A20 regulation of nuclear factor-kappaB: perspectives for inflammatory lung disease. Am J Respir Cell Mol Biol 2011; 44:743-8.

22. Vereecke L, Sze M, Mc Guire C, Rogiers B, Chu Y, Schmidt-Supprian M, et al. Enterocyte-specific A20 deficiency sensitizes to tumor necrosis factor-induced toxicity and experimental colitis. J Exp Med 2010; 207:1513-23.

23. Vereecke L, Beyaert R, van Loo G. The ubiquitin-editing enzyme A20 (TNFAIP3) is a central regulator of immunopathology. Trends Immunol 2009; 30:383-91.

24. Shembade N, Ma A, Harhaj EW. Inhibition of NF-kappaB signaling by A20 through disruption of ubiquitin enzyme complexes. Science 2010; 327:1135-9.

25. Das T, Chen Z, Hendriks RW, Kool M. A20/Tumor Necrosis Factor alpha-Induced Protein 3 in Immune Cells Controls Development of Autoinflammation and Autoimmunity: Lessons from Mouse Models. Front Immunol 2018; 9:104.

26. Ma A, Malynn BA. A20: linking a complex regulator of ubiquitylation to immunity and human disease. Nat Rev Immunol 2012; 12:774-85.

27. Zhou Q, Wang H, Schwartz DM, Stoffels M, Park YH, Zhang Y, et al. Loss-of-function mutations in TNFAIP3 leading to A20 haploinsufficiency cause an early-onset autoinflammatory disease. Nat Genet 2016; 48:67-73.

28. Wolfrum S, Teupser D, Tan M, Chen KY, Breslow JL. The protective effect of A20 on atherosclerosis in apolipoprotein E-deficient mice is associated with reduced expression of NF-kappaB target genes. Proc Natl Acad Sci U S A 2007; 104:18601-6.

29. Hsu AC, Dua K, Starkey MR, Haw TJ, Nair PM, Nichol K, et al. MicroRNA-125a and -b inhibit A20 and MAVS to promote inflammation and impair antiviral response in COPD. JCI Insight 2017; 2:e90443.

This article is protected by copyright. All rights reserved 
30. Vande Walle L, Van Opdenbosch N, Jacques P, Fossoul A, Verheugen E, Vogel P, et al. Negative regulation of the NLRP3 inflammasome by A20 protects against arthritis. Nature 2014; 512:69-73.

31. Kelly C, Williams MT, Mitchell K, Elborn JS, Ennis M, Schock BC. Expression of the nuclear factor-kappaB inhibitor A20 is altered in the cystic fibrosis epithelium. Eur Respir J 2013; 41:1315-23.

32. Li MY, Zhu M, Linghu EQ, Feng F, Zhu B, Wu C, et al. Interleukin-13 suppresses interleukin-10 via inhibiting A20 in peripheral B cells of patients with food allergy. Oncotarget 2016; 7:79914-24.

33. Liu X, Liu Y, Xu M, Li J, Teng X, Cheng H, et al. Zinc finger protein A20 is involved in the antipsoriatic effect of calcipotriol. Br J Dermatol 2016; 175:314-24.

34. Municio C, Dominguez-Soto A, Fuentelsaz-Romero S, Lamana A, Montes N, Cuevas VD, et al. Methotrexate limits inflammation through an A20-dependent cross-tolerance mechanism. Ann Rheum Dis 2018; 77:752-9.

35. Malcomson B, Wilson H, Veglia E, Thillaiyampalam G, Barsden R, Donegan S, et al. Connectivity mapping (ssCMap) to predict A20-inducing drugs and their antiinflammatory action in cystic fibrosis. Proc Natl Acad Sci U S A 2016; 113:E3725-34.

36. Sasse SK, Altonsy MO, Kadiyala V, Cao G, Panettieri RA, Jr., Gerber AN. Glucocorticoid and TNF signaling converge at A20 (TNFAIP3) to repress airway smooth muscle cytokine expression. Am J Physiol Lung Cell Mol Physiol 2016; 311:L421-32.

37. Altonsy MO, Mostafa MM, Gerber AN, Newton R. Long-acting beta2-agonists promote glucocorticoid-mediated repression of NF-kappaB by enhancing expression of the feedback regulator TNFAIP3. Am J Physiol Lung Cell Mol Physiol 2017; 312:L358-L70.

\section{Acknowledgements}

"The authors acknowledge financial support and supply of IRL201104 from Immune Regulation Ltd (formerly Peptinnovate Ltd), Stevenage, UK. We would also like to acknowledge Professor Chris Corrigan, recently retired Professor of Respiratory Medicine, King's College London for the provision of lung biopsies from patients with asthma and from healthy volunteers"

Conflict of interest: Professor Clive Page has received grants from Immune Regulation Ltd (formerly Peptinnovate Ltd) to support Dr Yanira Riffo Vasquez and to support the PhD studentship for Francis Man. Professor Page is also a non-Executive Director of Immune Regulation Ltd who are developing the peptide described in this work and he has equity in the Company. The other authors have no conflict of interest to declare 


\section{Legend of the Figures}

Fig. 1: Effect of IRL201104 on acute OVA-induced allergic inflammation in the lung. A, total cells in BAL fluid; B, eosinophils in BAL fluid; N left to right: 9, 13, 5, 4, 11. Mice were treated with IRL201104 intra-nasally 10 minutes before each OVA challenge (200, 20 and 2 ng/kg i.n). C, IL-5 levels in BAL of mice treated with $20 \mathrm{ng} / \mathrm{kg}$ of IRL201104; $\mathrm{N}$ left to right: 5, 8 and 5. D, Levels of IL-12 in BAL of mice treated with $20 \mathrm{ng} / \mathrm{kg}$ of IRL201104, N left to right: 5, 8 and 5. BAL collected $24 \mathrm{~h}$ after the 3rd OVA challenge. Data are expressed in mean + SEM and analysed using one way ANOVA with post hoc Dunnet's test. ${ }^{*} \mathrm{p}<0.01$, **p $<0.001 * * * * \mathrm{p}<0.0001$ vs OVA group, ${ }^{\alpha \alpha} \mathrm{p}<0.0001,{ }^{\alpha} \mathrm{p}<0.05$ vs sham group. $\mathbf{E}$, total cells in BAL fluid, $\mathrm{N}=5$. F, eosinophils in BAL fluid collected $24 \mathrm{~h}$ after the 3rd OVA challenge $\mathrm{N}=4$. Mice were treated with $20 \mathrm{ng} / \mathrm{kg}$ of IRL201104 intravenously 10 minutes before each OVA challenge. Data are expressed in mean + SEM and analysed using one way ANOVA with post hoc Tukey's test (E) and Bonferroni's test (F). ${ }^{* *} \mathrm{p}<0.001$ vs OVA group, ${ }^{\alpha} \mathrm{p}<0.001,{ }^{\alpha \alpha} \mathrm{p}<0.0001$ vs sham group. Data was obtained in 3 independent experiments.

Fig. 2: Effect of intravenous treatment with IRL201104 on acute HDM-induced allergic inflammation in the lungs of guinea pigs. A, eosinophils in BAL collected $24 \mathrm{~h}$ after the intra-tracheal challenge of $50 \mu \mathrm{g}$ HDM, N=6. B, extravasation of Evan's blue 1 hour after subcutaneous injection of a diluted solution of HDM, N=6. C. Lung resistance induced by $100 \mu \mathrm{g} / \mathrm{kg}$ of HDM i.v., N=6. Guinea pigs were treated with IRL201104 (200 ng/kg) intravenously 10 minutes before challenge with HDM. Data are expressed in mean + SEM, and analysed using unpaired T test with Welsh's correction (A and C) and two way ANOVA followed by Sidak's multiple comparisons test (B).***p<0.01 vs HDM group. Data was obtained in 2 independent experiments.

Fig. 3: Time course effect of IRL201104 on OVA-induced eosinophil infiltration into the lungs of allergic mice. Eosinophils in BAL collected $24 \mathrm{~h}$ (A), 10 (B), 20 (C) and 30 days (D) after last IRL201104 treatment. Mice were treated intravenously or intranasally with $20 \mathrm{ng} / \mathrm{kg}$ of IRL201104, 10 minutes before the first 3 OVA challenges only. Data are expressed as mean + SEM, and analysed using 
one way ANOVA with post hoc Dunnetts' test. ${ }^{*} \mathrm{p}<0.01,{ }^{*} \mathrm{p}<0.001$, vs OVA group, ${ }^{\alpha} \mathrm{p}<0.0001$ vs sham group. $\mathrm{N}=4$ /group. Data was obtained in one single experiment.

Fig. 4. Effect of post-treatment with IRL201104 on an established OVA-induced lung inflammation. Total number of cells (A) and eosinophils (B) in BAL collected $24 \mathrm{~h}$ after the 6th OVA challenge. Mice were treated intranasally with $200 \mathrm{ng} / \mathrm{kg}$ of IRL201104 24h after OVA challenge (one treatment) or 24,48 and $72 \mathrm{~h}$ after last OVA challenge ( 3 treatments). Challenges 4,5 and 6 were performed 10 days after challenge 3 and during this subsequent phase of allergen challenge no further treatment with IRL201104 was performed. Data are expressed as mean + SEM, and analysed using one way ANOVA with post hoc Dunnetts' test. ${ }^{\alpha} \mathrm{p}<0.001$ vs sham group $* * \mathrm{p}<0.001$, ***p $<0.0001$ vs respective OVA group control. N=5-6/group. Data was obtained in one single experiment.

Fig. 5: Effect of IRL201104 on the expression of A20 and NF- $\mathrm{AB}$ in lung tissue. A. Area of expression of A20, B. activated NF-kB (phospho S536) and C. total NF-kB (p65) in paraffin embedded mouse lung tissue. Lungs were collected 24 h, 10 days, 20 days and 30 days after IRL201104 treatment. Mice were treated intranasally with $20 \mathrm{ng} / \mathrm{kg}$ of IRL201104, 10 minutes before the first 3 OVA challenges only in a total of 3 treatments. Data are expressed as mean + SEM, and analyzed using one way ANOVA with post hoc Tukey's test. ${ }^{*} \mathrm{p}<0.01$, ***p $<0.0001$, vs OVA control group; $\mathrm{N}=4$ mice/group. Data was obtained in one IHC assay.

Fig. 6: Expression of $\mathbf{A} 20$ in human lung tissue and circulating leukocytes. A. Quantification of the regions of positive staining for A20 measured by immunohistochemistry. B. Percentage of expression of A20 measured by flow cytometry in circulating leukocytes collected from healthy and severe asthmatic patients. C. Representative photomicrograph of human lung tissue collected from healthy and mild steroid naïve asthmatic donors (20x magnification). D. Representative scatter plots and histograms of A20 expression from healthy (upper panel) and asthmatic donors (lower panel). Data are expressed as mean + SEM in graph B and as median with 95\% CI in graph C. Analyzed using unpaired $t$-test with post-hoc Mann Whitney test $* \mathrm{p}<0.05$ vs healthy, $* * \mathrm{p}<0.001$ vs healthy. $\mathrm{N}=3$ /group in graph $\mathrm{B}$, two

This article is protected by copyright. All rights reserved 
independent experiments. $\mathrm{N}=10$ healthy donors and 9 asthmatics in graph C. Data in graph B was obtained in one single IHC assay. Data in graph C was obtained in 5 independent flow cytometry measurements.

Fig. 7: Effect of IRL201104 on human circulating leukocytes. Whole blood was obtained from A. healthy or B. severe asthmatic donors and incubated for 24 hours with IRL201104 $(0.1-10 \mu \mathrm{g} / \mathrm{mL})$ or without (untr). After lysis of red cells and permeabilization, A20 expression in total blood leukocytes was measured by flow cytometry. Representative scatter plots and histograms of A20 expression from healthy C. and D. asthmatic donors untreated or treated with $0.1 \mu \mathrm{g} / \mathrm{ml}$ of IRL201104. FL4 is PerCP A20 label. Values represent median with 95\% confidence interval and was analyzed using one way ANOVA with post-hoc Dunnett's test * $\mathrm{p}<0.01,{ }^{*} \mathrm{p}<0.001$ vs unstimulated cells. $\mathrm{N}=8$ asthmatic donors and 11 healthy donors. Data was obtained in 10 independent flow cytometry measurements.

Fig. 8: Effect of IRL201104 on human ASM. Cells were isolated from healthy or mild asthmatic donors. Cells were cultured until reaching confluence and then incubated for 4 hours with IRL201104 alone (A and D), or in combination with $10 \mathrm{ng} / \mathrm{mL}$ of TNF- $\alpha$ (B and $\mathbf{E}$ ) or $100 \mathrm{ng} / \mathrm{mL}$ of LPS (C and F). A20 expression was measured by immune fluorescence and representative images of the intensity of the staining can be seen in the photographs taken with a 40x objective from healthy and asthmatic cells unstimulated and untreated (a and e), treated with $10 \mathrm{ng} / \mathrm{mL}$ of TNF- $\alpha$ for $4 \mathrm{~h}$ (b and f), 0.5 $\mathrm{pg} / \mathrm{mL}$ of IRL201104 for 4h alone (c and $\mathbf{g}$ ) or the combination of both (d and h). Values represent mean + SEM of background corrected integrated density and were analysed using one-way ANOVA with post hoc Tukey's test $* * * \mathrm{p}<0.0001$ vs unstimulated. ${ }^{* *} \mathrm{p}<0.001$ vs LPS or TNF- $\alpha . \mathrm{N}=3$ donors/group, two independent experiments. In the lower panel are representative photomicrographs of fluorescent cells. Data was obtained in 2 independent assays.

This article is protected by copyright. All rights reserved 


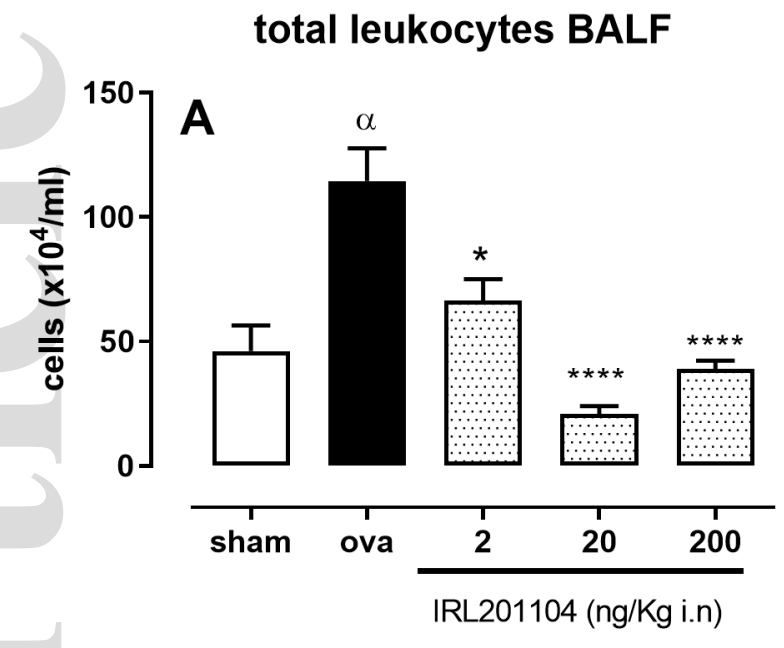

IL5

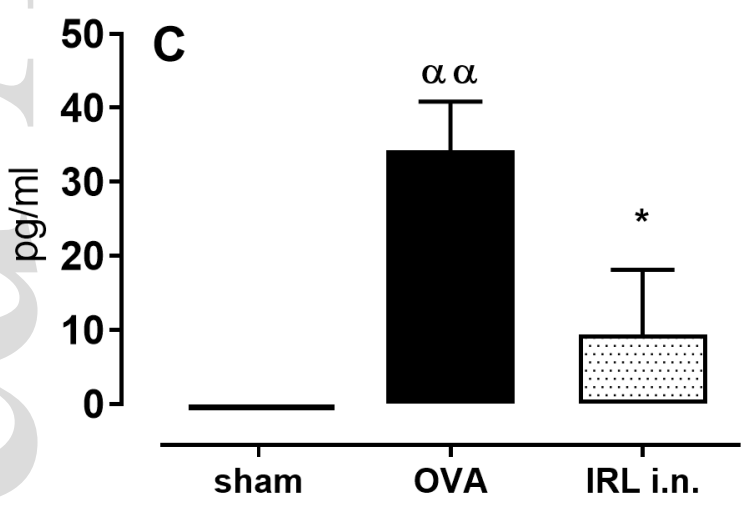

total leukocytes BALF

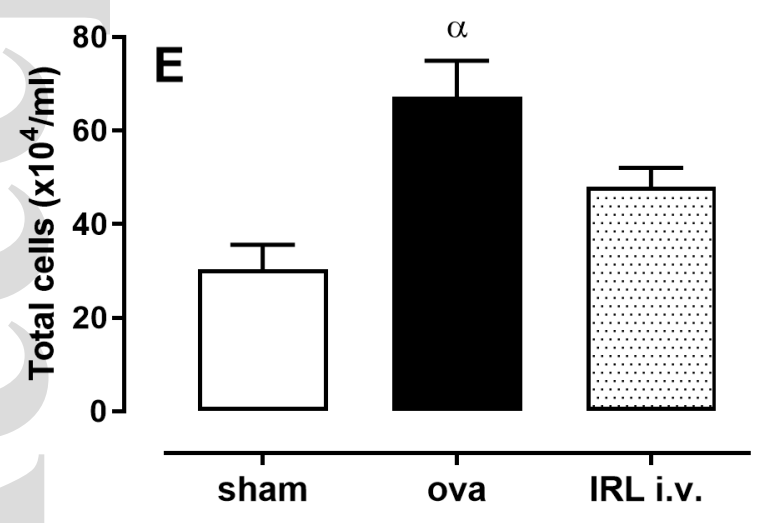

eosinophils

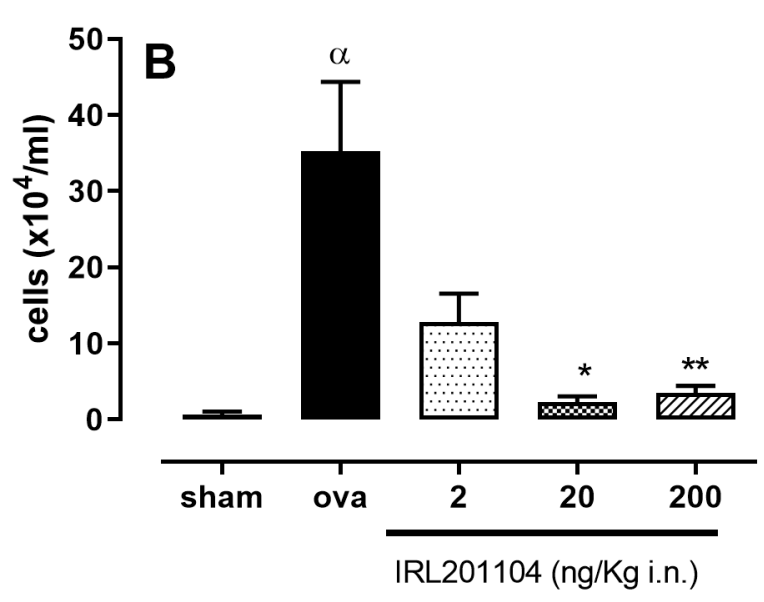

IL-12

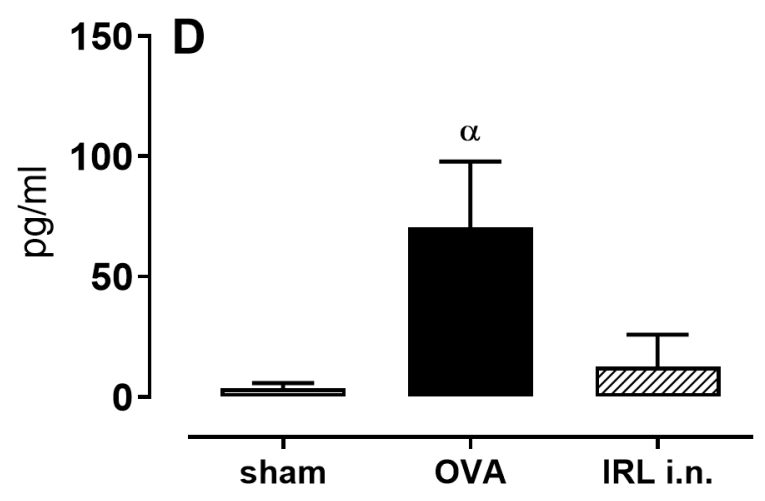

eosinophils

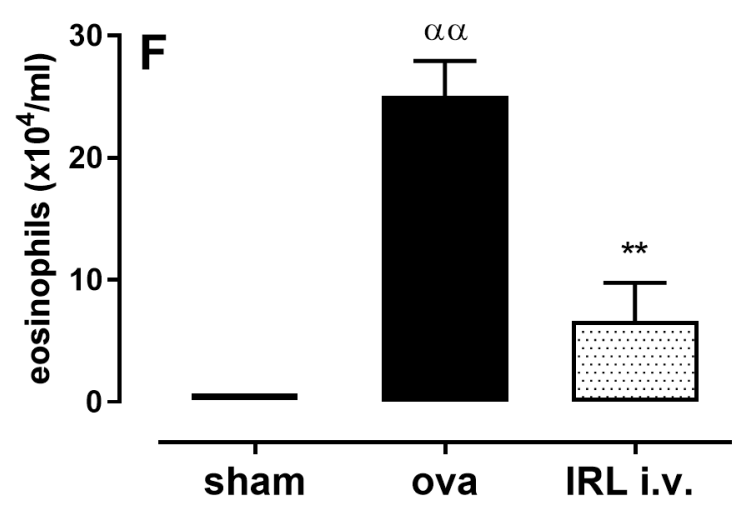

Fig.1 Riffo-Vasquez, Y. et al., 2019 

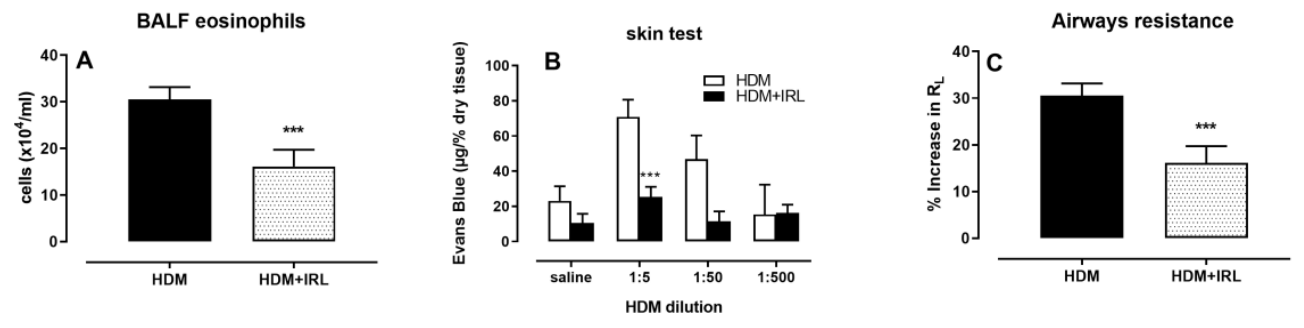

Fig. 2 Riffo-Vasquez, Y. et al, 2019

cea_13550_f2.tif 

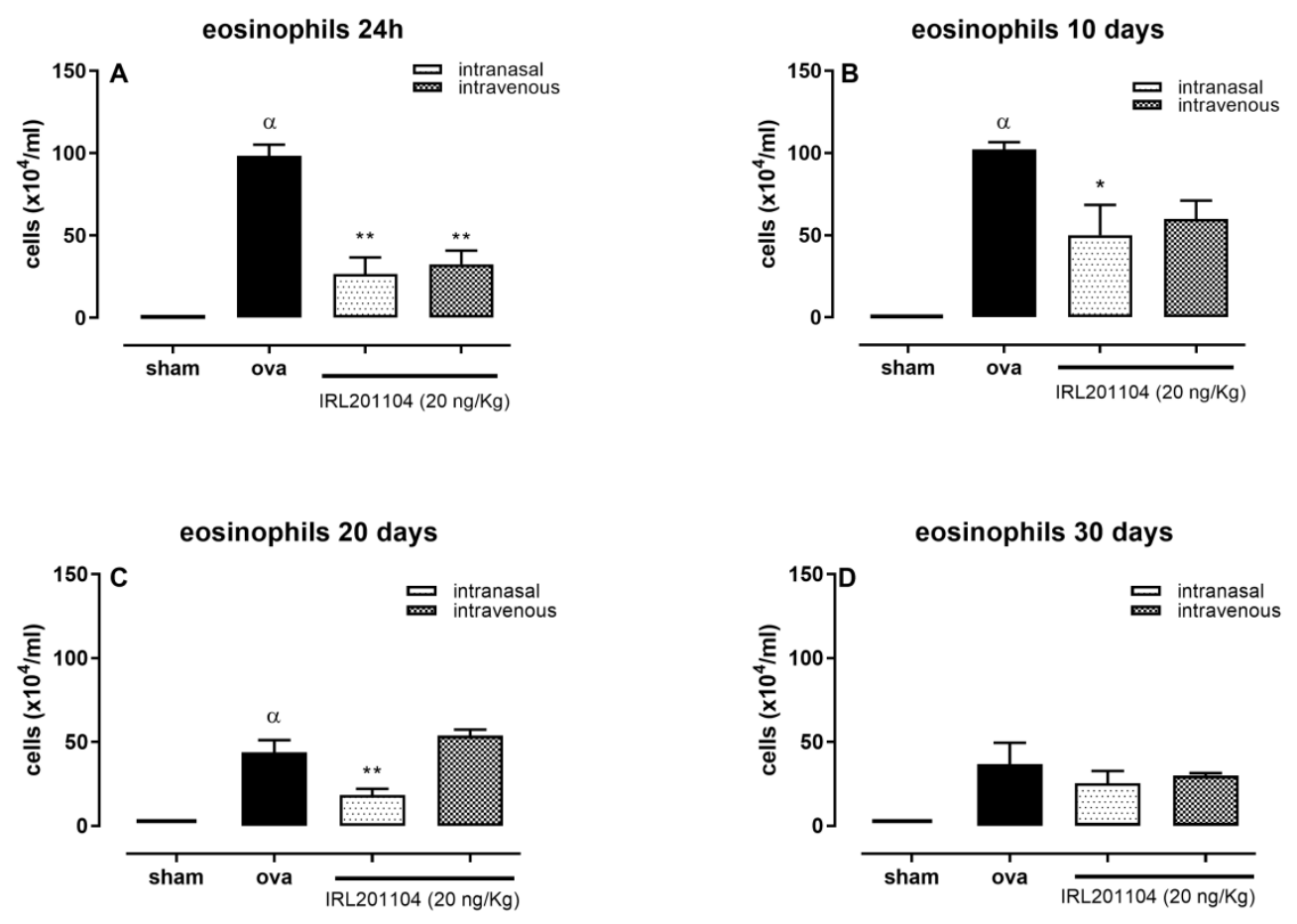

Fig. 3 Riffo-Vasquez, Y., 2019

cea_13550_f3.tif 

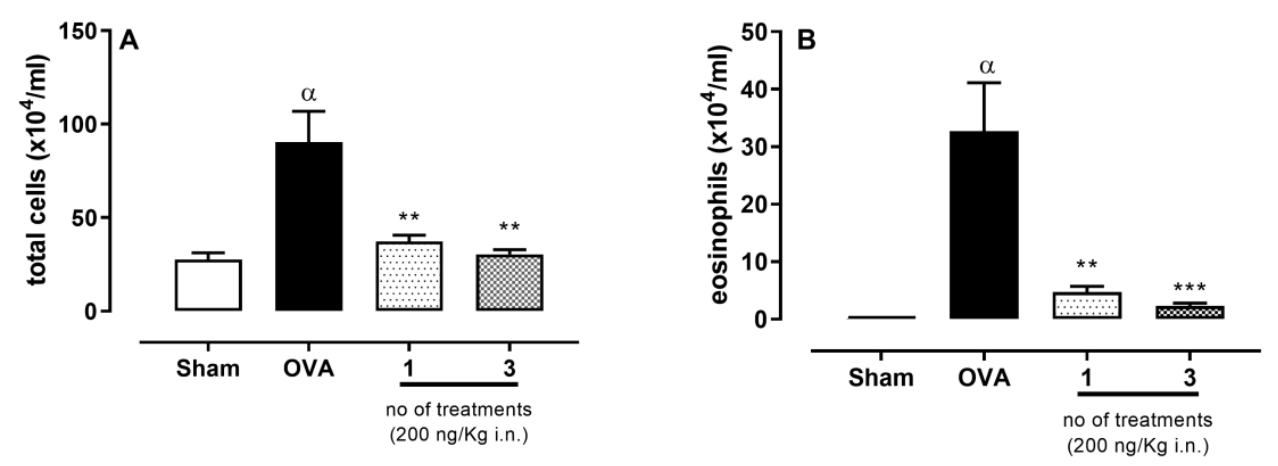

Figure 4 Riffo-Vasquez, Y. et al. 2018

cea_13550_f4.tif 

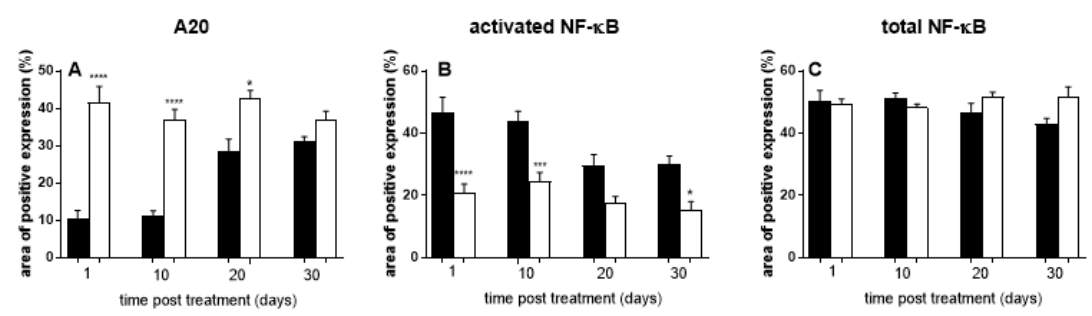

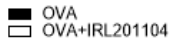
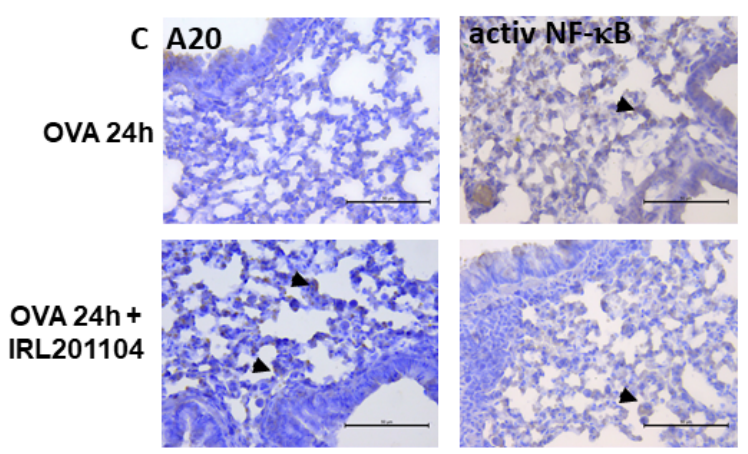

Total NF=kB

Figure 5 Riffo-Vasquez et al., 2019

cea_13550_f5.tif 

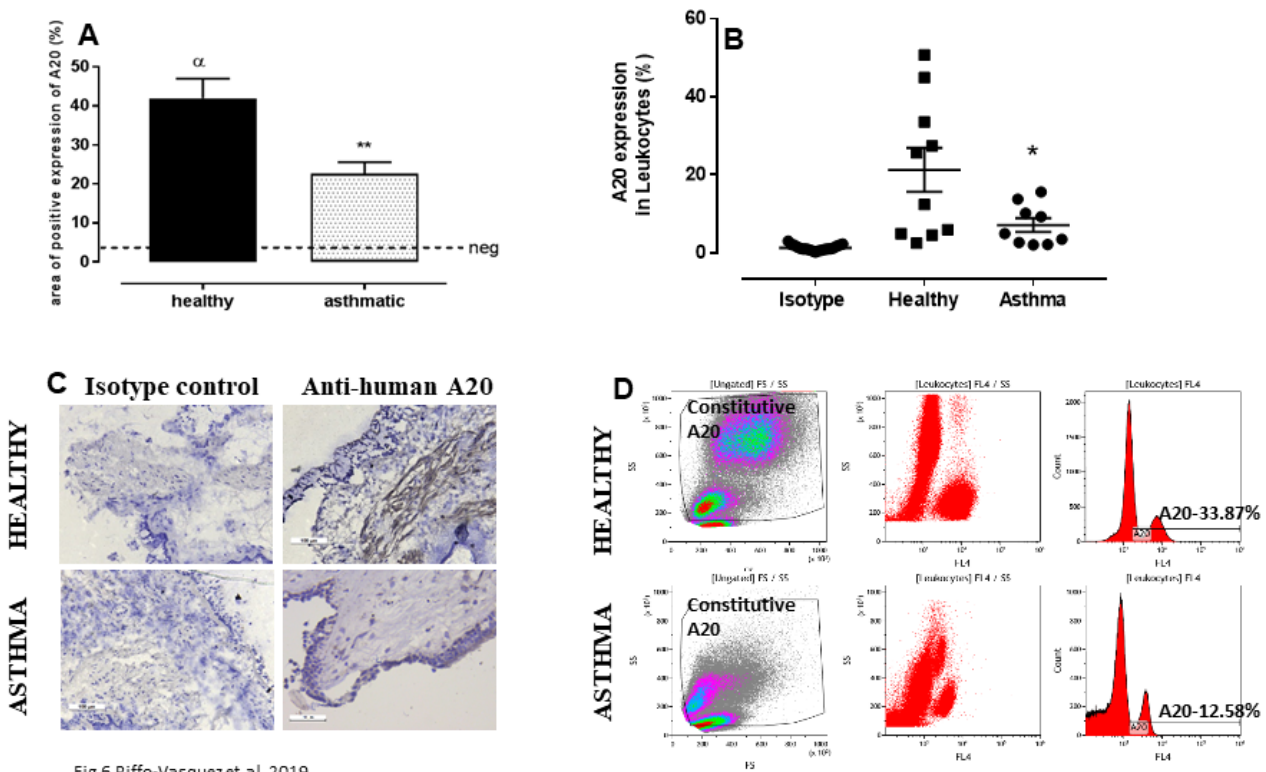

cea_13550_f6.tif 

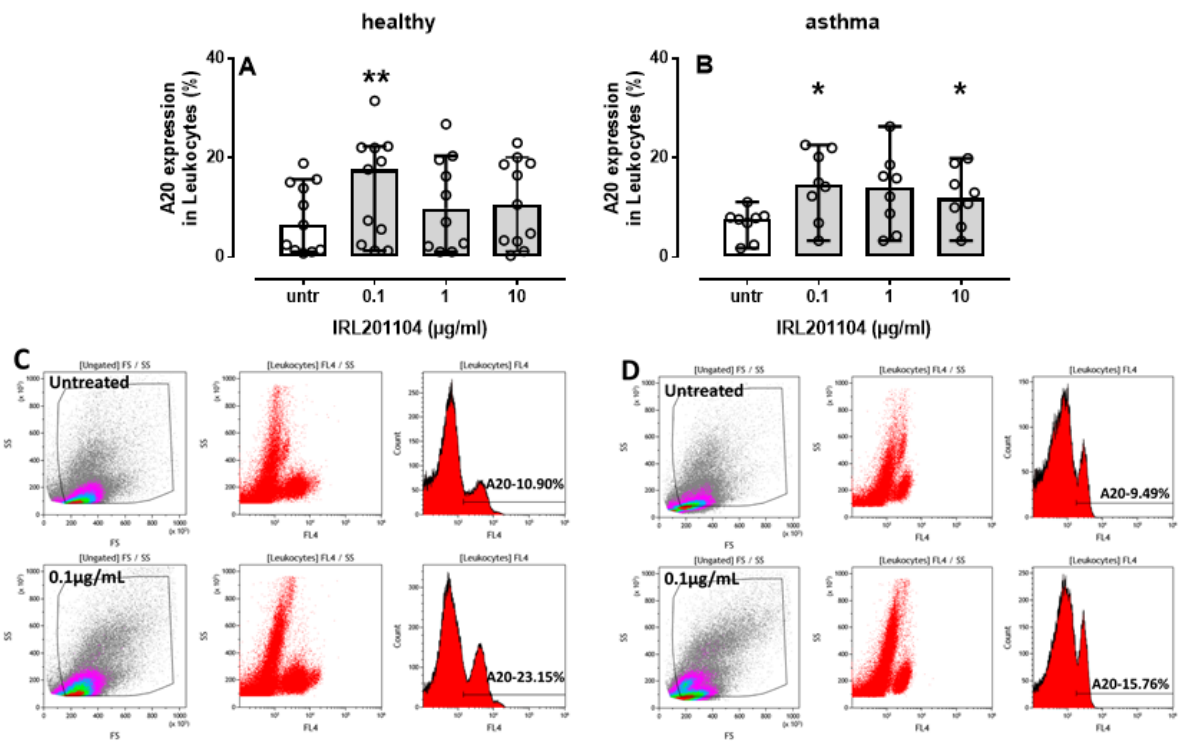

Figure 7. Riffo-Vasquez et al., 2019

cea_13550_f7.tif 

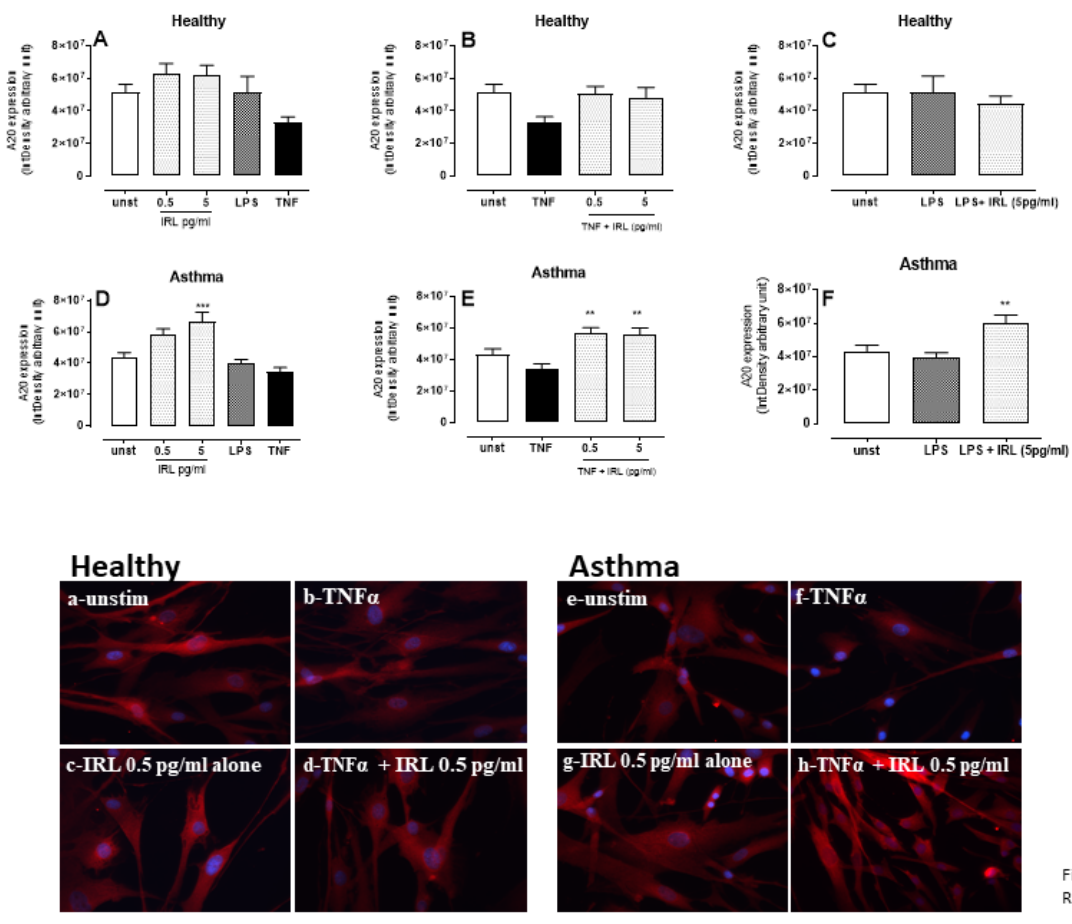

Fig. 8

Riffo-Vasquez, Y. 2019

cea_13550_f8.tif 